\title{
The subtle beauty of art in the service of science
}

An illustration may be intended to emphasize details, convey an idea or raise questions.

Sir - As a professional scientific illustrator I feel compelled to respond to Julio Ottino's Commentary "Is a picture worth 1,000 words?" (Nature 421, 474-476; 2003). I believe that Ottino's criticisms of scientific illustration are founded on an incorrect understanding of the field.

Galileo's drawings can't be compared with magazine covers: they are two unrelated types of illustration. It is incorrect to conclude from such a comparison that scientific disparity exists between them because the magazine covers "are left in the hands of artists and illustrators" - this artwork was intended to enhance editorial material rather than to illustrate research.

Such conceptual illustrations are designed to pose questions. Their use on the cover of a science magazine offers the promise of articles that inform these questions. The cover art of the 30 January 2003 issue of Nature (see figure) and the related News and Views and Letter (Nature 421, 489-490 \& 530-533; 2003) follow this convention. The image does not illustrate the research itself; that is not its intended application. Furthermore, the choice of digital medium, whether used by the hand of a scientist or the hand of an artist, has no bearing on this question.

Scientific illustration follows a different mandate, and it can often be found within the pages of the very magazines under

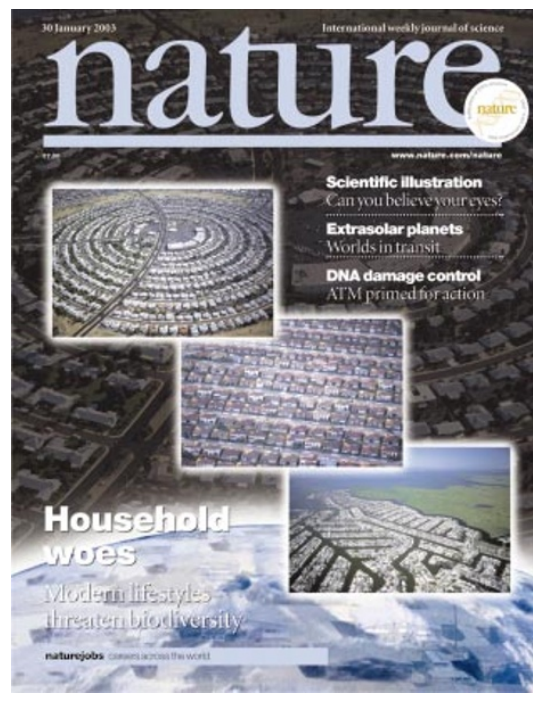

discussion. These drawings outline structure and clarify detail, as required by the subject and requested by the researcher. Because they communicate subtleties and eliminate the ambiguities of language, scientific illustrations are an important, often necessary, element in precise communication (see The Guild Handbook of Scientific Illustration, edited by E. R. S. Hodges; Van Nostrand Reinhold, 1989).

Scientific illustration is a clearly defined field that benefits from active collaboration between scientist and illustrator. Using their professional observational skills, scientific illustrators strive to render the most accurate representation of their subject. It is, by definition, art in the service of science. The act of drawing is, in essence, the act of editing. Complaints about omitted details miss this important point. Scientific illustrators are trained to eliminate non-essential information. The twisted stem of a dried plant is smoothed out. The broken edge of a fossil bone is repaired. Cracks and discoloration may be removed. These subjects are thus rendered in a way chosen to amplify those details that require emphasis.

Scientific illustrations, even conceptual cover art, should be as accurate as possible. However, Ottino's proposal to establish rules governing the use of realistic rendering techniques is superfluous. Professional standards are already in place for scientific illustration. Magazine editors recognize that their educated readership can distinguish between a beautifully rendered concept and the current state of scientific research. Scientific illustrations exist within this context. They communicate with and within conventions that reach back in time from this issue of Nature to the pages of Galileo's notebooks.

Frank Ippolito

Division of Vertebrate Paleontology, American Museum of Natural History, 79th Street $\&$ Central Park West, New York, New York 10024, USA

\section{No strings attached to \$225m sponsorship}

Sir — David Ritson in his Commentary "Fuel for thought" (Nature 421, 575-576; 2003), addressing Stanford's new Global Climate and Energy Project (G-CEP), inaccurately describes the motivations and arrangements for this programme.

The premise of G-CEP is that energy is a critical component of modern societies. Supplying energy for a growing world population while significantly reducing emissions of greenhouse gases is one of the grand challenges of this century. It is entirely appropriate for Stanford faculty and students, working with institutions around the world, to engage in research that will have global benefits.

Stanford's independence is fully protected. The project's sponsors have their own substantial, related research programmes. They have chosen also to support research in a university because it brings a healthy independence of views that they value and support. Academic freedom is an essential component of that, as both Stanford and G-CEP's sponsors agree. Stanford's considerable experience in working with companies, both in protecting the interests of research and in developing applications, will guide this project.

Any technologies developed under the G-CEP will be widely available, and all the work done will be reported publicly, as required by Stanford's standard policy on openness in research. Patents that arise from the research will be held by Stanford. During the first five years, a short period on the timescale of changes in energy systems, the sponsoring companies can license those technologies. Subsequently, Stanford and sponsors may license and sublicense broadly.

The G-CEP agreement explicitly calls for Stanford to explore broadly technology alternatives that can supply energy with substantial reductions in greenhouse emissions. There has been no attempt to favour fossil fuels or to exclude other energy sources. An initial G-CEP project involves research on the biological generation of hydrogen, for example.

The three-year funding commitments under G-CEP are less restrictive than many research support agreements. Federally funded research projects often make continued support contingent on availability of funds and acceptable progress; funding for existing projects can be delayed or cancelled if agency budgets are reduced.

Under G-CEP, the proposal cycle will be shorter and the reporting requirements less stringent, and there will be greater flexibility to pursue new ideas.

Charles Kruger*, Franklin M. Orr Jr $\dagger$ ${ }^{*}$ Vice Provost and Dean of Research, Building 18, Stanford University, Stanford, California 94305, USA $\dagger$ Director, Global Climate and Energy Project, Stanford University, Stanford, California 94305, USA

\section{correspondence}

Contributions to Correspondence may be submitted to corres@nature.com. They should be no longer than 500 words, and ideally shorter. Published contributions are edited. 